\title{
Signaling in leukemia: which messenger to kill?
}

\author{
Warren S. Pear
}

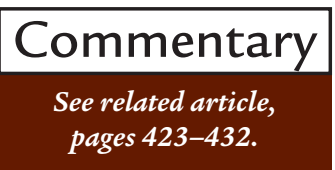

University of Pennsylvania, Department of Pathology and Laboratory Medicine and Institute for Medicine and Engineering, 611 Biomedical Research Building II/III, 421 Curie Boulevard, Philadelphia, Pennsylvania 19104-6160, USA. Phone: (215) 573-7764; Fax: (215) 573-8606;

E-mail: wpear@mail.med.upenn.edu.

Chromosomal translocations can activate oncogenes either by juxtaposing an inappropriate enhancer or promoter with a proto-oncogene or by fusing different genes to create novel combinations of protein-coding domains (1). The former mechanism is typical of lymphoid leukemias; in such cases, the regulatory region involved determines which cell types are at risk of transformation. For example, follicular lymphoma, a class of B-cell tumor, arises in cells containing at $(14 ; 18)$ chromosomal translocation that places the $B c l 2$ gene under the control of immunoglobulin regulatory sequences. When novel fusion proteins drive oncogenesis, on the other hand, cell specificity may have other sources: new activities resulting from gain or loss of specific sequences, protein relocalization leading to both gain and loss of substrate access, or modulation of activity by 1 of the fusion partners. Such fusion proteins are typically found in myeloid leukemias. For example, the $\mathrm{t}(8 ; 21)$ translocation creates the AML1/ETO fusion gene associated with the M2 subtype of acute myelogenous leukemia, and $t(9 ; 22)$ creates the $b c r / a b l$ oncogene which causes chronic myeloid leukemia. In this issue of the JCI, Tomasson et al. report an in vivo mutation analysis of another chimeric oncoprotein of this class, the TEL/PDGF $\beta R$ fusion protein found in a subset of patients with chronic myelomonocytic leukemia (2). These new data indicate that the oncotropicity of TEL/PDGF $\beta$ R depends critically on interactions between specific phosphotyrosine residues in the cytoplasmic portion of the oncoprotein, and a variety of signaling proteins containing the SH2 domain.

The TEL/PDGF $\beta$ R fusion protein was identified in leukemic cells from a patient with a variant of chronic myelomonocytic leukemia, which contained a $\mathrm{t}(5 ; 12)$ chromosomal translocation. The TEL/PDGF $\beta$ R fusion results in a predominantly cytoplasmic protein whose $\mathrm{NH}_{2}$-terminus is derived from the transcription factor TEL, but whose $\mathrm{COOH}$-terminus is contributed by PDGF $\beta R$ (3). TEL contributes an oligomerization domain to the fusion protein, allowing it to multimerize in the absence of ligand, which leads to constitutive PDGF $\beta R$ tyrosine kinase activity. Retained sequences from PDGF $\beta R$ include the transmembrane domain, the aforementioned tyrosine kinase domain, and tyrosines that serve as docking sites for proteins that mediate interactions with cytoplasmic signaling molecules. Lacking from the fusion protein are the DNA binding sequences of TEL and the ligand-binding sequences of PDGF $\beta$ R. Also, the reciprocal translocation product is not expressed. The oncogenic capacity of TEL/PDGF $\beta R$ has been revealed in both tissue culture and in vivo assays; it confers factor-independent growth on lymphoid and myeloid cells that otherwise require IL-3 to proliferate in culture, and it promotes the development of clonal lymphoid leukemias when expressed from a lymphoid-specific element in transgenic mice (3-5). The clonal nature of these tumors is significant, because it suggests that events in addition to TEL/PDGFßR expression are required to complete the process of transformation.

Tomasson et al. now show that TEL/PDGF $\beta$ R expression, driven by a retroviral long terminal repeat in murine hematopoietic progenitor cells, induces a leukemia resembling $\mathrm{t}(5 ; 12)$ chronic myelomonocytic leukemia. The murine TEL/PDGF $\beta$ R-induced disease is an aggressive and uniformly fatal myeloproliferative disease (MPD) (2). Because this disease is oligoclonal, TEL/PDGF $\beta$ R appears to be sufficient for leukemic induction. As might be anticipated, mutations that compromise either oligomerization or kinase activity abolish the transforming activities of the fusion gene (Figure 1). Tomasson and colleagues also generated a series of site-directed mutations in the fusion gene (Figure 1), and took advantage of the consistency and short latency period of the MPD in their system to test the roles of various intracellular tyrosine residues in pathogenesis. In choosing residues to mutate, they were guided by previous mutational analyses that had implicated specific $\mathrm{SH} 2$ domain-containing proteins in signaling by the native PDGF $\beta R$ (6).

Mutational dissection of TEL/PDGF $\beta R$. After ligand binding, the wild-type PDGF $\beta R$ protein dimerizes, activating the receptor's kinase domain to autophosphorylate 12 intracellular tyrosine residues (Figure 1), and recruiting a variety of $\mathrm{SH} 2$ domain-containing proteins to the receptor's cytoplasmic tail (6). These SH2-containing proteins do not bind indiscriminately, but recognize a 3-6 amino acid sequence immediately $\mathrm{COOH}$-terminal to a phosphotyrosine residue. The recruited proteins - Src family tyrosine kinases, adapter molecules such as GRB2, phosphatidylinositol 3-kinase, and transcription factors of the STAT family, among others - mediate a wide range of responses, including cell growth, adhesion, apoptosis, and motility.

Because interaction of SH2 domains with PDGF $\beta \mathrm{R}$ is dependent on tyrosine autophosphorylation, each of these interactions can be abrogated by introducing tyrosine to phenylalanine mutations at the different autophosphorylation sites. This approach allows a systematic dissection of the signaling pathways activated after ligand binding. For example, mutation of Y740 and Y751 in the wild-type PDGF $\beta \mathrm{R}$ protein (a change that abrogates PI3K binding) results in failure to protect PC12 cells from apoptosis, and a Y716F mutation, which abolishes GRB2 binding, inhibits activation of Ras in endothelial cells and fibroblasts $(6,7)$.

Based on previously described mutations in PDGF $\beta R$, Tomasson et al. generated 4 mutants in TEL/PDGF $\beta R$, targeting 2 or more tyrosine residues in each. They assessed the effects of these changes in culture and in vivo. 


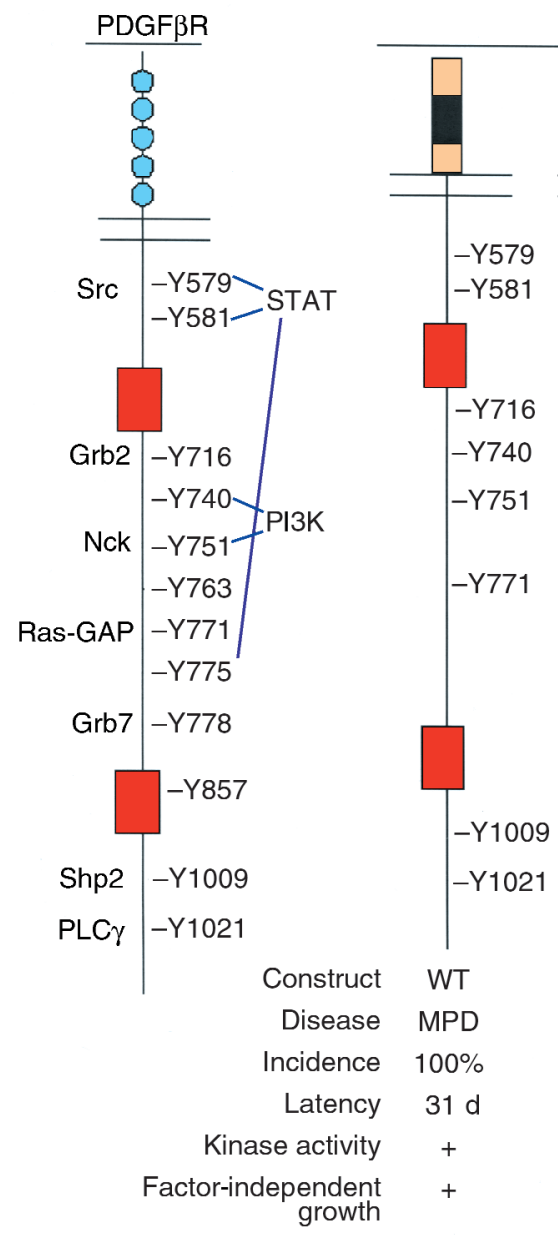

TEL-PDGF $\beta R$

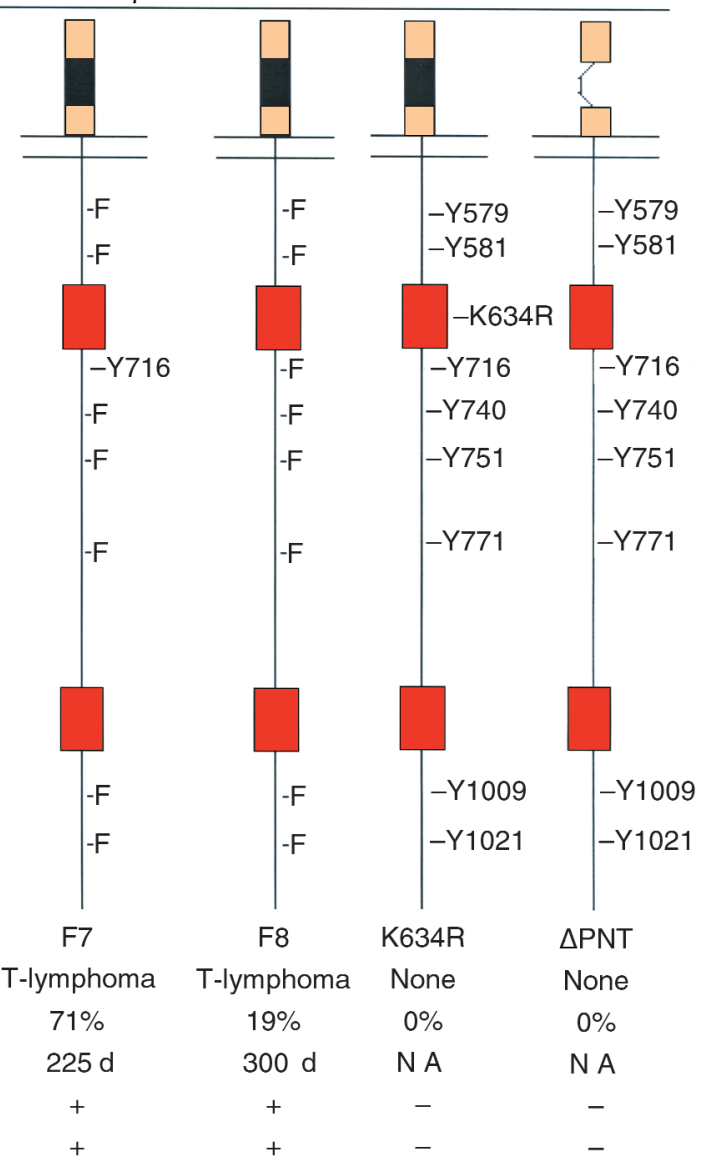

Figure 1

TEL/PDGF $\beta$ R leukemogenesis is influenced by phosphotyrosine interactions. At the left is 1 chain of PDGF $\beta$, showing tyrosine residues that become phosphorylated after ligand binding. The signaling complex is a dimer (only 1 chain is shown). Strongly interacting proteins are shown adjacent to the tyrosine residues to which they bind. As shown by STAT and PI3K, proteins can interact with multiple tyrosines. The right panels show TEL/PDGF$\beta R$ and the mutant proteins analyzed by Tomasson et al. (2). Only tyrosine residues that were mutated in their work are shown. The phenotypes of mice receiving the wild-type and mutant constructs are listed below each construct. " $\Delta \mathrm{PNT}$ " indicates deletion removing the pointed domain (indicated by the shaded box in TEL), which is necessary for oligomerization.

Although each mutant protein conferred factor-independent growth on IL-3-dependent cell lines, the leukemogenesis assay identified significant differences among the mutants. Mutation of the tyrosine residues that interact with PI3K (Y740, Y751), Nck (Y751), Ras-GAP (Y771), SHP-2 (Y1009), and PLC $\gamma$ (Y1021) had little effect on the phenotype of the MPD disease, but slowed disease onset. In contrast, mutation of Y579 and Y581, residues that are necessary for high-affinity interactions with Src-family kinases and STAT proteins, abolished the MPD; mice carrying proteins with these mutations (F2, F7, and F8; see Figure 1) succumbed instead to T-cell lymphoma.

Unlike the MPD conferred by the unaltered fusion protein, these T-cell lymphomas were clonal, suggesting that additional mutations are required to promote neoplasia. The phenotype of the T-cell lymphoma could be altered by additional tyrosine to phenylalanine mutations. The F7 mutant (which is predicted to abolish interactions with many of the known signaling molecules, although it leaves the GRB2 binding site intact) conferred a longer latency and decreased penetrance. The F8 mutant (which does not even interact with GRB2) increased the latency further, with fewer than $20 \%$ of the mice succumbing to T-cell lymphomas during the 300-day experimental period. Although these results suggest that additional mutations result in a graded effect on both latency and penetrance of the T-cell lymphomas, this general- ization should be confirmed by analysis of additional mutations; one approach would be targeting the 4 other phosphorylated tyrosines in the cytoplasmic tail of the fusion protein that were not assayed in the current analysis. The finding that the F8 mutant still conferred disease (unlike the K634R or $\triangle$ PNT mutants that inhibit tyrosine kinase activity) suggests that additional signaling pathways are induced by tyrosine phosphorylation and contribute to oncogenesis.

The simplest explanation of these results is that induction of a fatal MPD requires a constitutively active tyrosine kinase to induce Src- or STAT-dependent signaling. Both of these signaling pathways are important for transducing growth factor signals and for mito- 
genic and differentiation responses. The exact role that these signaling pathways play in myelopoiesis is unclear, but the MPD phenotype suggests that they play an essential role in regulating myeloid proliferation. When signaling through these pathways is blocked due to Y579F and Y581F mutations, myeloproliferation does not occur. However, outgrowth of other transformed populations does occur, consistent with the stem-cell nature of this leukemia. In all cases, T-cell lymphomas result, which probably reflects a bias in the murine model rather than limited transformation potential of TEL/PDGF $\beta R(5,8)$. Because the $\mathrm{T}$-cell disease develops relatively late in all cases (see Figure 1), lethal MPD may obscure the potential of the unaltered TEL/PDGF $\beta$ R to promote lymphoma. An alternative hypothesis to explain the switch in disease phenotype from MPD to T-cell lymphoma is that abrogation of signaling through Y579 and Y581 suppresses proliferation of the myeloid lineage while concurrently conferring neoplastic potential to T cells. Future studies, such as those discussed below, should discern between these possibilities.

The distinct disease phenotypes conferred by the various tyrosine mutants raises the compelling issue of differential gene expression in animals carrying the different oncogenic alleles. Fambrough et al. recently applied gene expression profiling using cDNA arrays to investigate the effects of PDGF signaling on the activation of immediate early gene expression in NIH 3T3 fibroblasts (9). Their study used mutants similar to those used by Tomasson et al., and showed that the major effect of the mutants was to affect the levels of gene expression rather than expression of specific sets of genes. This finding seems consistent with the observations by Tomasson et al. (2) of differences in disease latency or penetrance among the different alleles in the series. It appears less helpful in understanding the qualitative phenotypic differences between some alleles (for instance, between F2 and F5). However, it should be noted, first, that quantitative changes in gene expression can cause qualitative phenotypic differences if there is a sufficiently sharp threshold in the response to a given gene product, and second, that Fambrough et al. surveyed a limited set of cDNAs (9). Indeed, the critical genes that affect leukemogenesis in vivo may not be expressed in cultured cells such as NIH 3T3, and it is not obvious what cell type should best be studied to follow the onset of MPD. Work with the $b c r / a b l$ oncogene suggests that the murine disease targets a hematopoietic progenitor, but the identity of this progenitor is not known (8).

Parallels with $B C R / A B L$. The murine MPD induced by TEL/PDGF $\beta$ R is nearly identical to that induced by $\operatorname{BCR} / \operatorname{ABL}(8,10,11)$. The similarity in the induced murine diseases mirrors the clinical overlap in presentation of patients with chronic myeloid leukemia and $t(5 ; 12)$-associated disease (12). Both TEL/PDGF $\beta$ R and $\mathrm{BCR} / \mathrm{ABL}$ contain oligomerization domains and activated tyrosine kinases, and the paper by Tomasson et al. and analogous work on BCR/ABL mutants show that the parallels between these oncogenic fusion proteins extend still further. Thus, a tyrosine to phenylalanine mutation in $\mathrm{BCR} / \mathrm{ABL}$ residue 177, which mediates binding of GRB2, blocks transformation of fibroblasts but does not reduce the ability of BCR/ABL to confer factor independence to IL-3-dependent lymphoid cells $(13,14)$. A murine bone marrow transplant assay shows that this mutation, much like the F2 mutant described by Tomasson and colleagues, changes the nature of the oligoclonal MPD to a monoclonal Tcell lymphoma $(15,16)$. It will be of interest to assess the effect of a single site mutation in TEL/PDGF $\beta$ R tyrosine 716, which binds GRB2, to determine if this mutant is sufficient to change the phenotype of the disorder. Other mutations in BCR/ABL appear to affect the latency of the myeloproliferative disorder. For example, mutation or deletion of the $\mathrm{SH} 2$ domain slows disease onset in a fashion similar to that of the F5 mutation (X. Zhang et al., unpublished observations). Gene expression profiling should be helpful in understanding whether similar signaling pathways are activated by this mutation in $\mathrm{BCR} / \mathrm{ABL}$ and by the $\mathrm{F} 5$ variant of TEL/PDGFßR.

Studies with both TEL/PDGF $\beta$ R and $\mathrm{BCR} / \mathrm{ABL}$ confirm that the most effective way to abrogate disease is to eliminate the tyrosine kinase activity of either fusion protein. For this reason, there is great excitement about the use of the specific tyrosine kinase inhibitor
STI 571, which is showing efficacy in early clinical trials when used to treat patients with $\mathrm{BCR} / \mathrm{ABL}$-positive disease $(17,18)$. The alternative approach, targeting specific signaling molecules that act downstream of the kinase, appears to be inadequate to prevent leukemias that arise from hematopoietic stem cells. In the F8 mutant of TEL/PDGF $\beta R$, at least 8 different signaling interactions are lost, but T-cell lymphoma still occurs (albeit with reduced penetrance and slower onset). Moreover, any therapy that inhibited Src-, STAT-, and GRB2-mediated signaling would surely cause significant toxicity. Leukemias represented by $\mathrm{BCR} / \mathrm{ABL}$ and TEL/PDGF $\beta \mathrm{R}$ are not representative of all myeloid leukemias, and in vivo studies of the signaling pathways that lead to myeloid leukemia will surely uncover additional targets for drug development, such as proteins in the STAT5 signaling pathway, which have proved to be essential for in vivo transformation by the TEL/JAK2 fusion oncoprotein (19). Because the optimal therapeutic targets for each class of disease will generally depend on the rate-limiting oncogenic event, the analysis of disease pathways in relevant in vivo models will no doubt continue to guide rational drug design.

\section{Acknowledgments}

Thanks to R. Ren and R. van Etten for sharing unpublished results, and to $\mathrm{Y}$. He and J. Wertheim for critical reading of the manuscript. Work in the author's lab is supported by the National Institutes of Health and the Leukemia Society of America.

1. Rabbitts, T.H. 1994. Chromosomal translocations in human cancer. Nature. 372:143-149.

2. Tomasson, M.H., et al. 2000. Fatal myeloproliferation, induced in mice by TEL/PDGF $\beta$ R expression, depends on PDGF $\beta$ R tyrosines 579/581. J. Clin. Invest. 105: 423-432.

3. Golub, T.R. 1997. TEL gene rearrangements in myeloid malignancy. Hematol. Oncol. Clin. North Am. 11:1207-1220.

4. Carroll, M., Tomasson, M.H., Barker, G.F., Golub, T.R., and Gilliland, D.G. 1996. The TEL/plateletderived growth factor beta receptor (PDGF beta R) fusion in chronic myelomonocytic leukemia is a transforming protein that self-associates and activates PDGF beta R kinase-dependent signaling pathways. Proc. Natl. Acad. Sci. USA. 93: $14845-14850$

5. Tomasson, M.H., et al. 1999. TEL/PDGFbetaR induces hematologic malignancies in mice that respond to a specific tyrosine kinase inhibitor. Blood. 93:1707-1714.

6. Heldin, C.H., Ostman, A., and Ronnstrand, L. 1998. Signal transduction via platelet-derived growth factor receptors. Biochim. Biophys. Acta. 
1378:F79-F113.

7.Arvidsson, A.K., et al. 1994. Tyr-716 in the platelet-derived growth factor beta-receptor kinase insert is involved in GRB2 binding and Ras activation. Mol. Cell. Biol. 14:6715-6726.

8. Pear, W.S., et al. 1998. Efficient and rapid induction of a chronic myelogenous leukemia-like myeloproliferative disease in mice receiving P210 bcr/abl-transduced bone marrow. Blood. 92:3780-3792.

9. Fambrough, D., McClure, K., Kazlauskas, A., and Lander, E.S. 1999. Diverse signaling pathways activated by growth factor receptors induce broadly overlapping, rather than independent, sets of genes. Cell. 97:727-741.

10. Zhang, X., and Ren, R. 1998. Bcr-Abl efficiently induces a myeloproliferative disease and production of excess interleukin-3 and granulocytemacrophage colony-stimulating factor in mice: a novel model for chronic myelogenous leukemia. Blood. 92:3829-3840.

11. Li, S., Ilaria, R.L., Jr., Million, R.P., Daley, G.Q., and Van Etten, R.A. 1999. The P190, P210, and $\mathrm{P} 230$ forms of the BCR/ABL oncogene induce a similar chronic myeloid leukemia-like syndrome in mice but have different lymphoid leukemogenic activity. J. Exp. Med. 189:1399-1412.

12. Wessels, J.W., et al. 1993. t(5;12)(q31;p12). A clinical entity with features of both myeloid leukemia and chronic myelomonocytic leukemia. Cancer Genet. Cytogenet. 65:7-11.

13. Pendergast, A.M., et al. 1993. BCR-ABL-induced oncogenesis is mediated by direct interaction with the SH2 domain of the GRB-2 adaptor protein. Cell. 75:175-185.

14. Goga, A., McLaughlin, J., Afar, D.E., Saffran, D.C. and Witte, O.N. 1995. Alternative signals to RAS for hematopoietic transformation by the BCR-
ABL oncogene. Cell. 82:981-988.

15. Pear, W.S., et al. 1997. Murine models for studying the pathogenesis of chronic myelogenous leukemia. Blood. 90(Suppl.):393a.

16. Million, R.P., and Van Etten, R.A. 1999. The Grb2 binding site is required for induction of chronic myeloid leukemia-like disease in mice by the Bcr/Abl tyrosine kinase. Blood. 94(Suppl.):600a.

17. Druker, B.J., et al. 1999. Clinical efficacy and safety of an abl specific tyrosine kinase inhibitor as targeted therapy for chronic myelogenous leukemia. Blood. 94(Suppl.):368a.

18. Druker, B.J., and Lydon, N.B. 2000. Lessons learned from the development of an Abl tyrosine kinase inhibitor for chronic myelogenous leukemia. J. Clin. Invest. 105:3-7.

19. Schwaller, J., et al. 1999. Requirement for STAT5 for transformation by TEL/JAK2 but not BCR/ABL. Blood. 94(Suppl.):380a. 